\title{
RELEXIONS AU SUJET DE LA BLASTOGENĖSE
}

\author{
PAR \\ PAUL BRIEN \\ Université de Bruxelles
}

\section{SOMMAIRE}

I. Modalités du bourgeonnement des Ascidiacés . . . . . . . . . 47

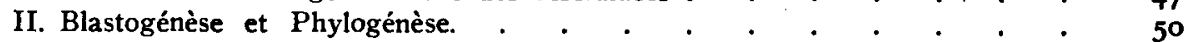

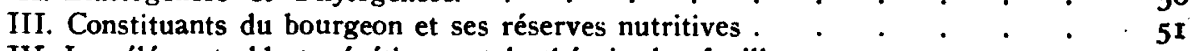

IV. Les éléments blastogénétiques et la théorie des feuillets . . . . . . . $\quad . \quad 5$ I

V. Organogénèse des bourgeons. Potentialités totales, Potentialités restreintes

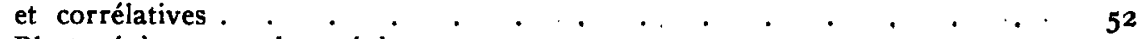

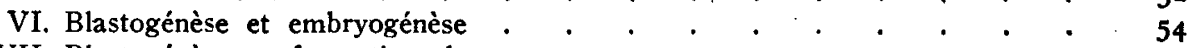

VII. Blastogénèse et formation du germen. . . . . . . . . . . . $\quad . \quad 55$

La reproduction assexuée n'a pas, dans l'ensemble du règne animal, la généralité de la reproduction sexuée. Elle n'en est pas moins un moyen de propagation de l'espèce, un aspect de l'ontogénèse des individus, la forme peut être la plus primitive de la multiplication à laquelle se sont superposées, indépendamment et au cours de l'évolution, les modalités diverses de la sexualité. Pour des raisons techniques, l'ontogénèse a été plus particulièrement et admirablement étudiée chez le zygote ou l'oeuf fécondé et chez l'embryon qui en dérive. Elle a apporté des notions importantes, des acquisitions fécondes dans les connaissances biologiques. Pour que les déductions qui en découlent et qui sont propres à la reproduction sexuée, puissent être généralisées à la physiologie du développement, elles doivent s'accorder aux processus blastogénétiques. C'est pourquoi il peut être utile de ramener l'attention sur ces derniers.

Il n'est guère possible dans le cadre d'un article de rappeler tous les cas de bourgeonnement qui se présentent dans le règne animal. Mais ils peuvent être résumés, en leur signification générale, à propos d'un groupe zoologique, celui des Tuniciers, où les manifestations blastogénétiques sont les plus étonnantes et plus diversifiées. Encore nous limiterons nous, en ce moment, aux Ascidiacés.

\section{MODALITES DU BOURGEONNEMENT DES ASCIDIACES}

Si l'on caractérise un mode de bourgeonnement par l'élément blastogénétique le plus important, on peut distinguer divers types de blastogénèse chez les Ascidiacés. 
I. Les A plousobranches.

a) Le bourgeonnement stolonial mésoblastique, statoblastique des Clavelinidae Les blastozoïdes se forment dans les chambres bourgeonnantes, dilatations variqueuses des stolons postabdominaux de tout ascidiozoïde. Ils y sont édifiés par un massif mésoblastique provenant de la condensation du septum mésenchymateux stolonial et par la portion ectodermique de la chambre bourgeonnante, coiffant ce massif blastogénétique. Celui-ci édifie tous les organes et tous les tissus autres que l'ectoderme du nouvel ascidiozoïde. Ce bourgeonnement mésoblastique stolonial est dit statoblastique parce que la chambre bourgeonnante s'isole du système stolonial (Clavelina lepadiformis), ce qui n'est peu être pas le cas pour les espèces dont les stolons restent enrobés dans une tunique commune (Clavelina phlegrea).

b) Le bourgeonnement post-abdominal épicardique ou abdominal entéro-épicardique des Polyclinidae

On sait en effet que le long postabdomen de tout ascidiozoïde des Polyclinidae, se strobilise en bourgeons selon l'axe antéro-postérieur de la souche.

Or, ce postabdomen contient, outre le tractus ovotestis, le prolongement de l'épicarde qui y refoule à l'extrémité distale l'organe cardio-péricardique. C'est le tronçon d'épicarde, cette fois, qui, dans chaque strobile, édifie tous les organes, à l'exception de l'ectoderme et des éléments génitaux. Dans un genre Sydnium (Aplidium) zostericola, au moment de la blastogénèse, l'abdomen s'allonge et se confond avec le postabdomen. C'est donc tout l'abdomen avec son contenu (épicarde, anse digestive, tractus génital) qui se strobilise. C'est à dire que les deux tronçons de l'anse digestive collaborent avec l'ép:carde. Celui-ci n'aura plus à assumer la formation du tube digestif dans l'édification du blastozoïde.

\section{c) Le bourgeonnement stolonial épicardique larvaire des Polycitoridae}

Ce bourgeonnement est ainsi dénommé par ce que l'on en connait chez Distaplia. Dans la larve, avant son éclosion, l'épicande gauche s'introduit dans une hernie ectodermique ventrale, hernie qui s'isole dans la tunique pour devenir un probourgeon. Ce probourgeon est rendu responsable de la formation de la multitude des blastozoïdes de la même colonie par strobilisation successive. Les blastozoïdes eux-mêmes ne sont plus capables de bourgeonnement, mais deviennent sexués. Il y aurait donc alternance des deux modes de reproduction, l'oozoïde fondateur étant exclusivement blastogénétique, łes blastozoïdes exclusivement sexués.

On connait des espèces de Distaplia et de Colella ou de tels bourgeons se rencontrent au sein de la colonne. Chez Colella ils y sont rares mais très. longs, et à strobilisation active à l'une de leurs extrémités.

Dans un travail récent, BERRIL reconnait cependant aux blastozoïdes, la possibilité d'émettre des bourgeons, lorsqu'ils sont sur le point de mourir. A ce. 
moment, la portion abdominale se condense, s'isole, devient un „épicardium" qui projette alors une sphérule blastogénétique ( $D$. Bermudensis). Il y a tout lieu d'admettre l'observation de BERRIL. Elle mériterait cependant d'être l'objet d'une étude histologique afin de déceler la véritable nature des éléments de cette blastogénèse secondaire de Polycitoridae.

\section{d) Le bourgeonnement double entéro-épicardique des Didemnidae}

Un mềme Ascidiozoïde, (oozoïde ou blastozoïde) de Didemnidae forme deux bourgeons, l'un thoracique, l'autre abdominal, qui sont complétifs l'un de l'autre. Le bourgeon thoracique comprend le pharynx, les cavités péribranchiales, le système nerveux, l'oesophage, l'atrium et l'extrémité rectale, le tout édifié par deux ampoules émises, chacune respectivement, par chacun des deux épicardes de la souche. Le bourgeon abdominal par contre, comprend une anse digestive, l'organe cardiopéricardique, deux épicardes et la masse génitale. Il est certain que l'anse digestive du bourgeon abdominal dérive de l'oesophage de la souche. Nous avons pu vérifier également que l'organe cardiopéricardique et les deux épicardes qui l'accompagnent dans le bourgeon abdominal, dérivent des deux tubes épicardiques de la souche. Il en résulte que ces deux bourgeons ont tous les organes pour se compléter l'un l'autre en un ascidiozoïde typique. Le bourgeonnement y est donc bien double et entéro-éricardique.

\section{Les Phlébobranches.}

\section{a) Le bourgeonnement abdominal entéro-épicardique des Diazonidae}

Dans cette famille qui, par ses caractères morphologiques, est intermédiaire entre les Aplousobranches et les Phlébobranches proprement dits, les Ascidiozoïdes sont coloniaux; les abdomens sont inclus dans une tunique commune. En hiver, ils s'amputent de leur thorax. Les abdomens par contre sont capables de régénérer les thorax nouveaux ou de se fragmenter en tronçons régénérant de nouveaux Ascidiozoïdes. La régénération est réalisée par l'épicarde et par les fragments d'anse digestive. Le bourgeonnement peut donc être appelé entéro-épicardique.

\section{b) Le bourgeonnement stolonial, mésoblastique, colonia! des Pérophoridae}

Les Péropharidae sont nettement des Phlébobranches. L'anse digestive est rejetée sur le flanc de l'animal. Il n'y a plus d'épicarde, par contre des stolons relient tous les ascidiozoïdes les uns aux autres en colonies traçantes et ramifiées. Pérophora retrouve ainsi, avec une structure différente, les conditions éthologiques approximativement comparables à celles des Clavelinidae. Les bourgeons s'édifient, de po:nt en point, à l'extrémité du stolon en croissance et aux dépens d'un massif mésoblastique provenant de la condensation du mésenchyme septal. Le bourgeonnement y est donc stolonial, mésoblastique, colonial. 
3. Les Stolidobranches

\section{Le bourgeonnement palléal des Polystyelinae et des Botryllidae}

Les Polystyelinae et les Botryllidoe, si voisins les uns des autres, ont le même processus de blastogénèse, avec des modalités distinctes. Ils sont constitués par tout ascidiozoïde (oozoïde, ou blastozoïde), aux dépens d'expansions latérales de l'ectoderme, expansions où s'introduit un diverticule du feuillet externe de la cavité péribranchiale.

\section{BLASTOGENESE ET PHYLOGENESE}

Dans tous les cas, le bourgeon étant un fragment de la souche, les éléments qui le constituent dépendent de la structure de cette dernière. Il en résulte que les modalités de la blastogénèse sont conditionnées par la phylogénèse.

Exception faite pour les Clavelinidae, l'épicande est l'élément blastogénétique essentiel dans les divers modes de bourgeonnement des Aplousobranches. Par leur anatomie et leur embryologie les Polycitoridae, les Polyclinidae et les Didemnidae sont des formes évoluées dont l'origine s'explique à partir des Clavelinidae, plus généralisées et plus primitives. On peut donc admettre que le bourgeonnement stolonial mésoblastique des Clavelinitae est primordial, tandis que les divers bourgeonnements épicardiques des Aplousobranches sont secondaires, conditionnés par les particularités de développement et de structure acquises au cours de l'évolution.

Chez les Polyclinidae, les Polycitoridae, les Didemnidae, les potentialités blastogénétiques de l'épicarde correspondent exactement aux possibilités régénératives virtuelles que cet organe possède chez les Clavelinidae. Si l'on sectionne une Claveline au niveau de la région oesophagienne il s'y reconstitue un nouveau thorax exclusivement aux dépens de l'épicarde. Dans le phylum des Aplousobranches la regénération devance et prépare la blastogénèse.

Parmi les Phlébobranches, les Diazonitae, pourvus d'un épicarde, sont seuls à présenter encore une modalité du bourgeonnement épicardique. Les Pérophoridae, par contre, privées d'épicarde reviennent à un bourgeonnement mésoblastique stolonial apparemment semblable à celui des Clavelinidae et corrélatif au retour des vaisseaux du test à l'état des stolons libres. Mais l'évolution est irréversible; le bourgeonnement des Pérophoridae manifeste des particularités qui le différencient de celui des Clavelinidae. Entre autres choses, il est stolonial et non statoblastique. L'organogénèse y est différente. On ne peut confondre, comme on l'a fait si souvent, ces deux modes de bourgeonnements mésoblastiques et stoloniaux, phylogénétiquement distincts.

Pour la même raison le bourgeonnement épicardique disparait chez les Stolidobranches. Dans ce groupe, des formes primitives, mais non blastogénétiques sont capables de régénération, c'est le feuillet péribranchial externe qui est l'élément essentiel de la régénération (Hétérocarpa: DE SÉlys Longchamps). Lorsque le bourgeonnement apparaît chez les Polystyelinae et chez 
les Botryllidae qui dérivent des premières, c'est cet élement qui devient blastogénétique; le bourgeonnement $y$ est palléal (péribranchial).

Les diverses modalités du bourgeonnement des Ascidiacés correspondent respectivement aux diverses étapes évolutives de leur structure. La blastogénèse est parallèle à la phylogénèse. Lorsque la blastogénèse apparait dans un groupe, elle extériorise naturellement les possibilités régénératives virtuelles des espèces non blastogénétiques et plus primitives; elle se réalise selon les mêmes processus organogénétiques et aux dépens des mêmes tissus de la souche. Dans la phylogénèse d'un groupe, la régénération devance la blastogénèse.

\section{CONSTITUANTS DU BOURGEON ET SES RESERVES NUTRITIVES}

Quelles que soient ses modalités, le bourgeonnement aboutit, par des moyens divers, au même résultat: le bourgeon est essentiellement constitué de deux vésicules emboîtées, l'externe ectoblastique, l'interne endoblastique, entre lesquelles sont interposés des éléments mésoblastiques. Sans doute, il peut s'y ajouter d'autres ébauches: ovotestis, cordon neural, tronçon d'anse digestive. C'est dans son mésenchyme haemocoelien que le bourgeon trouve la nourriture qui lui permet de grandir et de se développer. Si le bourgeon est attaché à la souche, son mésenchyme haemocoelien reçoit en dérivation le courant sanguin de celle-ci. Le blastozoïde s'en nourrit (Pérophoridae-Botryllidae). Le plus souvent, les bourgeons s'isolent mais auparavent des éléments sanguins, leucocytes à inclusions ou trophocytes venus de la souche, se sont accumulés dans son mésenchyme par un véritable phénomène d'embolie inflammatoire: chambre bourgeonnante des Clavelinidae, strobiles des Polyclinidae. Il se constitute un pseudo-vitellus qui sera histolysé et digéré au cours de la croissance et de l'organogénèse du blastozoïde. Entre ces deux types de bourgeons, les uns vivipares, si l'on peut dire, entretenus par la mère, les autres pseudo-vitellins, il existe des formes intermédiaires. Chez les Ditemnidae, les bourgeons se séparent de leur progéniteur quand leur développement est achevé et lorsqu'ils peuvent vivre par leurs propres moyens. D'autre part les stolons prolifères des Polycitoridae et les bourgeons qui en dérivent vivent détachés de l'oozoïde sans pseudo-vitellus, trouvant sans doute dans la tunique où ils sont enrobés, comme dans un mésenchyme périphérique, une nourriture diffuse suffisante.

\section{LES ELEMENTS BLASTOGENETIQUES ET LA THEORIE DES FEUILLETS}

Lorsqu'il entre en organogénèse, le bourgeon est donc formé de deux Vésicules emboitées, ecto- et endoblastique; entre lesquels se dispose un mésenchyme mésoblastique.

L'ectoblaste formera l'ectoderme, l'endoblaste, la plupart des organes, le 
mésoblaste constituera le mésenchyme haemocoelien, les muscles et les éléments génitaux (bourgeon de Clavelinidae, de Polyclinilae, de Polycitoridae). La vésicule endoblastique est donc l'élément organogénétique le plus important: il forme le pharynx, les cavités péribranchiales, le système nerveux et l'anse digestive, l'organe cardio-péricardique. Or, cette vésicule endoblastique prend sa source, selon les modalités du bourgeonnement, à partir des tissus de la souche les plus divers. Elle est d'origine mésoblastique chez les Clavelinidae, endoblastique chez les Polyclinidae, Polycitoridae, ectoblastique chez les Polystyélinae et les Botryllidae, c'est à dire qu'elle peut dériver de l'un des trois feuillets typiques de l'adulte.

Dans la blastogénèse, l'origine des éléments blastogénétiques à partir de l'un ou l'autre des trois feuillets n'a aucune signification. Les feuillets n'ont pas de spécificité organogénétique définitive ni absolue. Ce qui importe dans tous les cas, c'est que les éléments blastogénétiques, quelle que soit leur origine, reprennent l'état embryonnaire pour être l'ébauche organogénétique à potentialités multiples. Or cet état embryonnaire se manifeste par le fait que les cellules de l'ébauche blastogénétique d'où qu'elles proviennent, grossissent, acquièrent un cytoplasme basophile, s'enrichissent en acides ribonucléiques; leur noyau devient plus clair et est pourvu d'un nucléole bien marqué. Enfin ces cellules sont en prolifération intense.

Il ressort de ce qui précède qu'il n'y a pas de réserve blastogénétique qui se maintiendrait de génération en génération, continue et immortelle, comme on l'a prétendu jadis. Dans chaque Ascidiozoïde bourgeonnant, il existe une zone de croissance où les tissus restent ou redeviennent embryonnaires, s'enrichissent en substances basophiles réacquièrent le pouvoir de prolifération et d'organogénèse, sous l'action de facteurs actuels physiologiques qu'il conviendrait d'analyser en fonction de la physiologie générale de l'individu souche. Cette zone ainsi proliférante et organogénétique, correspondrait dans l'ensemble de l'organogénèse, à une sorte ,d'isolement physiologique”.

\section{ORGANOGENESE DES BOURGEONS. POTENTIALITES TOTALES, POTENTIALITÉS RESTREINTES ET CORRELATIVES}

On peut distinguer trois types d'organogénèse à partir de la vésicule endoblastique du bourgeon, celui du bourgeon des Clavelinidae celui des bourgeons des Polyclinidae, Polycitoridae et des Dimennidae (fort semblables à l'organogénèse de la régénération du tronçon abdominal de Claveline à partir de l'épicarde), celui des Pérophoridae qui se rapproche d'ailleurs des processus organogénétiques des Polystyelinae et des Botryllidae.

Sans rappeler les modalités de ces trois types d'organogénèse, il convient de dire qu'il implique au préalable l'existence d'une polarité. Le bourgeon est polarisé. Sa polarisation s'implante de novo (dans le bourgeon de Clave- 
linitae), elle est imposée par la polarité de la souche et par les connexions du bourgeon avec cette dernière chez les autres Ascidiacés.

L'organogénèse est une épigénèse dont les inductions, les corrélations évidentes sont loin cependant d'avoir été analysées expérimentalement. Si l'on s'en réfère à l'origine des éléments blastogénétiques fondamentaux, on constate que le mésenchyme de la souche intervenant dans la constitution de l'ébauche endoblastique du bourgeon de Clavelina et Pérophora est totipotent. Il édifie la totalité des organes du blastozoïde. Dans les cas où la vésicule endoblastique du bourgeon est formée d'emblée, soit par l'épicarde, soit par le feuillet péribranchial, cette vésicule supplante l'activité du mésenchyme qui se borne à former le mésenchyme haemocoelien, les muscles et parfois les éléments génitaux du blastozoìde.

D'autre part, le pouvoir organogénétique de la vésicule endoblastique se restreint dans la mesure ou d'autres ébauches blastogénétiques interviennent [cordon génital ovotestis du strobile des Polyclinidae; fragment d'anse digestive du strobile d'Aplidium zostericola ou dans le bourgeonnement des Didemnidae].

Rien ne fait' mieux saisir combien l'organogénèse du blastozoïde est le résultat des actions réciproques que les ébauches exercent les unes sur les autres. Il s'établit entre elles, sous l'influence de la polarité, des inductions corrélatives qui suscitent ou inhibent leur activité, de façon à maintenir l'unité morphologique de l'ensemble. A cet égard les potentialités de l'épicarde méritent d'être rappelées.

Dans un bourgeon de Polyclinidae, provenant de la strobilisation de son postabdomen, le tronçon d'épicarde reconstitue, à l'extrémité antérieure de l'axe de polarité du bourgeon, le pharynx, les cavités péripharyngiennes, le système nerveux, "anse digestive; à l'extrémité postérieure il forme l'organe cardiopéricardique. Ainsi la polarité du bourgeon règle son pouvoir organogénétique. Il est total à l'extrémité antérieure, il est restreint à l'extrémité postérieure mais d'une façon complétive. On peut affirmer que la formation cardiopéricardique est une potentialité restreinte inhibée puisque, si l'on envisage la chaine des bourgeons, on constate qu'au niveau d'un même étranglement, l'épicarde ne forme que l'organe cardiopéricardique à l'extrémité postérieure d'un bourgeon, tandis qu'il manifeste, à l'extrémité antérieure du bourgeon, suivant, sa potentialité la plus étendue en formant le thorax. Ceci se trouve confirmé par l'hétéromorphose de la régénération expérimentale d'un fragment d'abdomen de Claveline, fragment sectionné d'une part sous le thorax, de l'autre au dessus de l'estomac. Ces deux sections synchrones ont supprimé la polarité organogénétique dans ce fragment isolé. A chacune de ses extrémités, l'épicarde manifeste sa potentialité la plus étendue, il régénère un thorax. Or cette hétéromorphose n'a pas lieu si les deux sections sont successives, si le fragment oesophagien est isolé du thorax, 72 heures avant de l'être de la région stomodéale. Dans ce cas la régénération antérieure a eu le temps de 
s'amorcer, d'imposer une polarité au fragment; elle reforme un nouveau thorax avec tous ses organes. L'extrémité postérieure, capable elle aussi de former un thorax, restreint sa potentialité d'une façon complétive, en constituant ce qui manque: un organe cardiopéricardique.

Il en est de mềme dans le bourgeonnement des Didemnidae. Dans le bourgeon thoracique, l'épicarde est la seule ébauche blastogénétique. Elle édifie tout le thorax (potentialité totale). Dans le bourgeon abdominal, elle est en corrélation avec l'anse digestive, elle ne forme plus que l'organe cardiopéricardique.

On pourrait ajouter les cas des bourgeons où, à côté de l'épicarde, se trouvent d'autres ébauches, des tronçons d'anse digestive par exemple. A ceux-ci incombe de reformer l'anse digestive du blastozoïde. Les fonctions de l'épicarde en sont réduites d'autant. Quoiqu'il en soit capable, il n'intervient plus dans l'édification de cet organe puisque celle-ci est assurée par d'autres éléments. L'épicarde présente donc des dégrés dans l'extériorisation de ses possibilités régénératives. Il nous offre, dans le cas de la régénération des Aplousobranches, un exemple d'un élément embryogénétique aux possibilités variables, totales ou restreintes, mais toujours corrélatives selon les nécessités du développement de l'organisme et ajustées à ses besoins physiologiques.

\section{BLASTOGENESE ET EMBRYOGENESE}

Si l'on compare les développements embryonnaire et blastogénétique, on peut souligner quelques similitudes. L'oeuf et le bourgeon sont polarisés; ils ont une symétrie bilatérale, ils sont plus ou moins pourvus de réserves nutritives; ils sont hétérogènes êt constitués d'éléments plus ou moins spécifiques ou nombreux. Mais entre l'un et l'autre, il apparait aussi des différences fondamentales dans les processus même du développement.

Le bourgeon est immédiatement à un stade comparable à celui de la gastrula. Son développement est direct. Il évolue en ascidiozoïde, sans passer par des stades larvaires, sans récapitulation ancestrale. L'oeuf parait donc plus riche en possibilités organogénétiques, puisqu'il suit les détours héréditaires larvaires et ancestraux qui aboutissent à la formation du tétard, détours que la métamorphose corrige ensuite pour ramener l'organisme à la structure normale et définitive.

En dépit de cette première divergence, le développement blastogénétique et le développement embryonnaire arrivent, en dernière analyse à former des ascidiozoïdes identiques. Le fait est d'autant plus remarquable qu'ils suivent des voies absolument différentes. Par exemple, le coeur de l'oozoïde est constitué par deux expansions latéro-ventrales du mésoblaste primaire et représente une formation coelomique de l'oozoïde. Il dérive de la vésicule endoblastique dans le bourgeon.

Le tube neural suit dans l'oeuf, les processus organogénétiques de celui des 
Cordés et dérive de la plaque neurale pré-blastoporale. Dans le bourgeon il provient soit du mésoblaste (Clavelinidae), soit de l'endoblaste (bourgeon palléal et bourgeon épicardique).

Les cavités péribranchiales de l'embryon se forment par deux invaginations ectodermiques; elles sont toujours édifiées par la vésicule endoblastique du bourgeon, etc. etc.

Si remarquable que soit donc la spécificité des plasmes dans l'oeuf d'un Tunicier, on constate qu'elle ne se maintient plus chez l'ascidiozoïde adulte, puisque ce dernier produit des bourgeons où les tissus récupèrent des potentialités organogénétiques tout autres sans cesser de former cependant un animal identique. C'est à dire que l'analyse du mécanisme de l'organogénèse faite sur l'oeuf et l'embryon ne peut donc révéler qu'un aspect particulier des processus par lesquels se constitue un ascidiozoïde adulte.

\section{BLASTOGENÈSE ET FORMATION DU GERMEN}

Les blastozoïdes étant toujours sexués, leur formation entraine celle des glandes génitales. Il est des cas où les gonocytes sont transmis au bourgeon par la souche elle-même. Les strobiles des Polyclinidae emportent chacun un tronçon de l'ovotestis qui prolonge dans le postabdomen l'ovaire de la mère. Dans cette famille, il y a donc continuité du germen au sens le plus strict, entre tous les ascidiozoïdes d'une même colonie, comme il y a continuité de l'endoderme, de l'ectoderme, et du mésenchyme.

Chez les Botryllidae les auteurs ont parlé du passage par l'afflux sanguin, du progéniteur à ses bourgeons, de cellules germinales libres et isolées. Mais dans la plupart des cas, il y a néoformation du germen (Clavelinidae, Polycitoridae, Polystyelinae). Dans le stolon prolifère des Polycitoridae, il existe un tractus germinal médio-dorsal (Colella, Distaplia). Il est formé par l'endoblaste chez Distaplia magnilarva et un fragment passe en chacun de ses bourgeons. Il est vrai que ce cordon médio-dorsal peut être différencié en gonocytes avant que ne se détachent les bourgeons (Distaplia stylifera, Colella cerebriformis) Chez les Polystyelinae et chez les Clavenidae, les éléments génitaux se forment à partir des cellules indifférenciées du mésenchyme introduit dans les bourgeons.

Il. n'existe donc, dans ces diverses espèces, aucune continuité du tissu germinal entre ascidiozoïde et ses blastozoïdes. Le tissu germinal est néoformé dans le bourgeon. Cette observation se confirme expérimentalement lorsque, dans un animal susceptible de régénération, on enlève les organes sexués et le stock des cellules germinales qu'il possède. Le régénérat réacquiert son appareil sexuel complet (Hydroïde, Oligochète).

Or, dans la blastogénèse aussi bien que dans les cas de régénération, ce sont les éléments somatiques qui, après avoir repris un état embryonnaire, dans les conditions rappelées précédemment, reconstituent tout l'organisme et son nou- 
veau germen. Ce fait évident s'oppose aux conceptions classiques de Nussbaum et de Weismann.

Il n'y a point de distinction irréductible entre le soma et le germen, l'évolution du germen en soma est parfaitement réversible en certains cas.

D'autre part la néoformation du germen, dans les bourgeons aussi bien que dans les régénérats, démontre, en même temps, que la notion métaphysique de la continuité du germen est inutile et incorrecte, elle ne correspond pas à la réalité. La différenciation des cellules germinales est discontinue tout comme l'est celle des autres tissus de l'organisme. Elle n'est pas prédéterminée, mais déterminée par l'action des facteurs actuels de la physiologie embryonnaire. Elle est comme tout autre organe, la conséquence inéluctable du développement, que celui ci soit ontogénétique, blastogénétique ou régénérateur.

Les particularités des cellules germinales, bien distinctes il est vrai, et qui leur donnent après leurs différenciations une certaine autonomie, s'acquièrent en fonction de la physiologie embryonnaire. Le ,déterminant germinal" qu'on leur attribue est une propriété acquise au cours du développement, et selon des processus qui ne sont pas sans une certaine similitude avec ceux selon lesquels des cellules de la souche deviennent blastogénétiques. Dans les deux cas, le début de la différenciation est localisée dans l'organisme; elle se caractérise par un accroissement de taille, une basophilie cytoplasmique correspondant sans doute à un enrichissement en acides ribonucléiques, par une prolifération très intense, du moins au début, enfin par une sorte d'indépendance „d'isolement physiologique", vis à vis de l'organisme qui les porte et les nourrit.

Dans un massif embryonnaire, quelle que soit l'origine de celui-ci, les lignées de cellules reproductrices sexuelles, tout comme les autres lignées de somatocytes, apparaissents sous l'induction des corrélations organogénétiques, en leur place, en leur temps, en conséquence mềme de la cro:ssance. Il n'y a pas de dualité chez les Métazoaires.

\section{BIBLIOGRAPHIE}

Les indications bibliographiques figurent dans les Traités Généraux:

Grassé, P., I948. Traité de Zoologie, Vol. XI: Procordés, Masson, Paris.

Kukenthal et Krumbach, 1935-1937. Handbuch der Zoologie, Tunicata, Berlin-Leipzig. BronN's Thier Reich, I893-1911, Tunicata.

KORChelt et HeIDER, I895-1910. Lehrbuch der Vergleichende Entwicklungsgeschichte des Wirbellosen Thieres (Korchelt 1936), Jena.

Signalons encore à propos de cette question:

Berril, N. J., 1948. Budding and the Reproductive Cycle of Distaplia. The Quarterly Journal of Microsc. Science, vol. 89, Part 3.

Brien, P. et P. van DEN Breede, ig48. Róle de l'épicarde dans le bourgeonnement des Didernnidae. Bull. Classe des Sciences de l'Acad. Royale de Belgique, tome XXXIV.

Brien, P., 1948. Potentialités totales, Potentialités restreintes et corrélatives d'une même ébauche, à propos de la régénération de l'épicarde des Tuniciers. Bull. Classe des Sciences de l'Acad. Royale de Belgique, tome XXXIV. 